\title{
Translocator protein (TSPO): the new story of the old protein in neuroinflammation
}

\author{
Younghwan Lee $e^{1}$, Youngjin Park ${ }^{1}$, Hyeri Nam ${ }^{1}, \mathrm{Ji}_{\text {-Won Lee }}{ }^{1} \mathcal{E}$ Seong-Woon Yu ${ }^{1.2, *}$ \\ ${ }^{1}$ Department of Brain and Cognitive Sciences, Daegu Gyeongbuk Institute of Science and Technology (DGIST), Daegu 42988, \\ ${ }^{2}$ Neurometabolomics Research Center, Daegu Gyeongbuk Institute of Science and Technology (DGIST), Daegu 42988, Korea
}

Translocator protein (TSPO), also known as peripheral benzodiazepine receptor, is a transmembrane protein located on the outer mitochondria membrane (OMM) and mainly expressed in glial cells in the brain. Because of the close correlation of its expression level with neuropathology and therapeutic efficacies of several TSPO binding ligands under many neurological conditions, TSPO has been regarded as both biomarker and therapeutic target, and the biological functions of TSPO have been a major research focus. However, recent genetic studies with animal and cellular models revealed unexpected results contrary to the anticipated biological importance of TSPO and cast doubt on the action modes of the TSPO-binding drugs. In this review, we summarize recent controversial findings on the discrepancy between pharmacological and genetic studies of TSPO and suggest some future direction to understand this old and mysterious protein. [BMB Reports 2020; 53(1): 20-27]

\section{TRANSLOCATOR PROTEIN (TSPO) AS A MULTI-FACETED MITOCHONDRIAL PROTEIN}

Mitochondria serve as the powerhouses of the cell by generating ATP coupled with respiratory oxidative phosphorylation of the nutrients. Mitochondria have other essential roles, including metabolite biogenesis, $\mathrm{Ca}^{2+}$ homeostasis, reactive oxygen species (ROS) production, inflammation and immunity, and programmed cell death $(1,2)$. Therefore, mitochondria are the crucial integrator of vital and detrimental cellular processes, and dysfunction of mitochondria is intimately implicated in a variety of neurodegenerative and neurological conditions, including Alzheimer's disease (AD), Parkinson's disease (PD), Huntington's disease (HD), anxiety, and bipolar disorder (3).

${ }^{*}$ Corresponding author. Tel: +82-53-785-6113; Fax: +82-53-7851209; E-mail: yusw@dgist.ac.kr

https://doi.org/10.5483/BMBRep.2020.53.1.273

Received 22 October 2019

Keywords: Microglia, Mitochondria, Neuroinflammation, Steroidogenesis, TSPO
Mitochondrial membrane proteins are the important regulators of mitochondrial homeostasis, such as ion transport, ATP/ADP transport, and mitochondria fusion/fission. Therefore, defects in these proteins are associated with numerous diseases (4). For this reason, mitochondrial membrane proteins are considered to be promising targets for development of diagnostic tools and therapeutic strategies. TSPO is the mitochondrial transmembrane protein that was discovered as the binding site of benzodiazepine in 1977 (5). Studies using TSPO-binding ligands have revealed that this protein participates in a variety of cellular functions, including cholesterol transport and steroid hormone synthesis, mitochondrial permeability transition pore (mPTP) opening, mitochondrial respiration, apoptosis, proliferation, tumorigenesis, and inflammation (6-10). In the brain, because of a noticeable increase in the protein expression level of TSPO in activated microglia during neuroinflammation and marked immunosuppressive potency of the TSPO ligands, TSPO has become an attractive pharmacological target for diagnostic imaging and anti-inflammatory, neuroprotective therapeutic design for the treatment of neurological and neuropsychological diseases (11).

\section{Evolution of TSPO}

TSPO has 169 amino-acid residues with highly conserved five transmembrane domains (TM) (Fig.1A). During evolution, the TSPO gene family has expanded its roles from environmental sensor to functional bioregulator (12). In bacteria, TSPO homologues serve as a regulator of photosynthesis and oxygen sensor (13). Even though the mammalian TSPOs share less than $30 \%$ identity in protein sequence with bacterial homologues, mammalian TSPO can replace bacterial TSPO's function as an oxygen sensor (13). Recently, TSPO in non-photosynthetic eubacterium Pseudomonas fluorescens was shown to share close structural and functional similarities with mammalian TSPOs including binding affinity to PK11195, a prototypical TSPO ligand (14). TSPO is also found in some populations of archaea and plants (12). However, absence of TSPO is also reported in Escherichia coli and yeast Saccharomyces cerevisiae, indicating that TSPO is not a necessary protein for biological activity or that other proteins can replace TSPO's function in some organisms (15). Animals and plants have more than one TSPO gene, namely, Tspo1 and Tspo2 (16). Tspo2 emerged by 
A
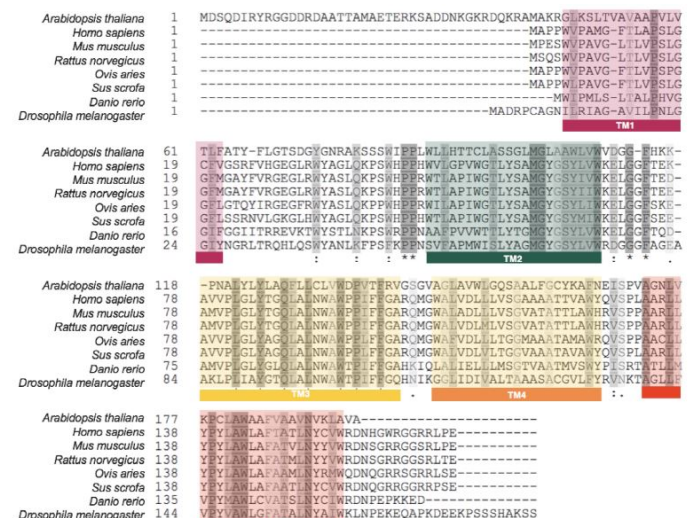

.
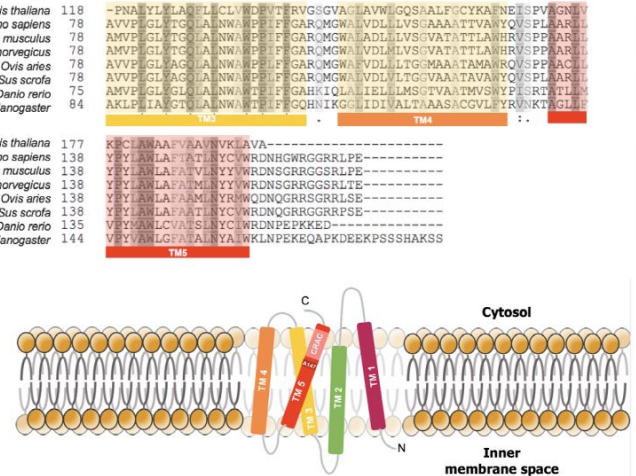

Fig. 1. Sequence alignment of eukaryotic TSPO homologues. (A) TSPO has a highly conserved sequence, especially on transmembrane domains from plants to human. (B) TSPO topology in the mitochondrial membrane.

gene duplication, which occurred before the divergence of the avians and mammals. Comparative analysis of TSPO1 and TSPO2 revealed that TSPO2 had lost its ligand-binding affinity. The subcellular location of TSPO2 is also different from the mitochondrial location of TSPO1, since TSPO2 is located on ER and nuclear membranes with limited distribution specific to hematopoietic tissue and erythroids (12). These evolutionary findings helped to understand the myriad functions of the TSPO family.

\section{Structure of TSPO}

The three-dimensional high-resolution structure of TSPO has been solved for mammalian or bacterial TSPO proteins (17-19), in which 5 TMs of TSPO are tightly packed together in the clockwise order TM1-TM2-TM5-TM4-TM3 when viewed from the cytosol (Fig. 1B) (18). Several metabolites and peptides, including cholesterol, porphyrins, phospholipase A2, and diazepam-binding inhibitor, can bind to TSPO, suggesting the presence of the endogenous TSPO ligands (20). The cholesterol recognition amino-acid consensus (CRAC) site starting with the amino-acid residue Ala 147 is found in TM5 and, together with the charged C-terminal end, faces toward the cytosolic side. This C-terminal region of TSPO plays an important regulatory role during cholesterol binding and import into mitochondria (17). PK11195 does not bind to CRAC, but binds to the pocket that is formed by the five transmembrane helices in the upper cytosolic part of the helical bundle (18).
PK11195 binding stabilizes the structure of TSPO, which explains the stimulation of cholesterol transport into mitochondria by this synthetic ligand [19]. Ala 147 residue is mutated to Thr in the polymorphism associated with increased anxiety in humans (21). Interestingly, this residue is involved in binding to both cholesterol and PK11195, and binding affinity of both ligands is decreased in the human Ala147to-Thr TSPO mutant (17). A proposed model of bacterial TSPO mimicking human polymorphism revealed differences in structure and conformational changes upon ligand binding, especially around the CRAC site, and provided insights into the potential pathogenic mechanism of TSPO polymorphism linked to psychiatric diseases in humans. Therefore, decreased binding affinity of ligands to mutated TSPO may underlie the pathogenesis of human psychiatric disorders, which warrants more in-depth future study to understand the pathophysiological roles of TSPO in the brain.

\section{Functions of TSPO}

The best-known function of TSPO is steroidogenesis (6). Steroidogenesis starts with cholesterol as the substrate, which is cleaved by the cholesterol side-chain cleavage enzyme, cytochrome P450 family 11, subfamily A member 1 (CYP11A1), located in the inner mitochondrial membrane (IMM). Sidechain cleavage by CYP11A1 produces pregneolone, the precursor of all steroids, leading to the synthesis of steroid hormones through a complex process of steroidogenesis (22).

In adrenocortical and Leydig tumor cell lines, which have highly steroidogenic activities, TSPO ligands promote steroid hormone production $(23,24)$. On the other hand, TSPO knockdown or disruption with homologous recombination in rat Leydig tumor cells reduced steroid hormone production $(25,26)$. Therefore, TSPO is considered to mediate the transport of cholesterol into IMM and play an essential role in steroidogenesis.

Another function ascribed to TSPO is regulation of $\mathrm{mPTP}$ opening (27); mPTP is formed by the assembly of the voltagedependent anion channel (VDAC) and adenine nucleotide transporter (ANT) as key components. Opening of this pore increases the permeability of mitochondria and allows the moving of molecules with a molecular weight $<1.5 \mathrm{kDa}$ through the membrane. Opening of mPTP uncouples oxidative phosphorylation and ATP synthesis, leading to energy depletion, loss of mitochondrial membrane potential (MMP), generation of mitochondrial ROS, and release of pro-cell-death proteins, which eventually lead to programmed cell death $(28,29)$. PK11195 alone at a high concentration can accelerate mPTP opening induced by $\mathrm{Ca}^{2+}$ overloading, a process in which VDAC participates. A low concentration of PK also accelerated opening of MPTP in synergistically with the VDAC inhibitor (30). These biochemical analyses suggest that TSPO as a partner of VDAC in modulation of $\mathrm{mPTP}$, and a VDAC/ANT/TSPO model was suggested to explain the effects of TSPO ligands (31). However, recent genetic studies called into question the 
pivotal roles of TSPO in steroidogenesis and mPTP (32), as will be discussed in Section 3.

\section{THE ROLES OF TSPO IN THE NERVOUS SYSTEM}

In the central nervous system (CNS), TSPO is highly expressed in glial cells (e.g., astrocytes, microglia), endothelial cells, tanycyites, and some populations of neurons (33-35). For decades, some studies have used TSPO ligands to assess the levels of neuroinflammation and microglia activation and to understand the pathophysiological roles of TSPO in the brain. However, understanding of the TSPO function in the brain has faltered, partly owing to lack of studies employing cell-type specific approaches in normal and pathological states.

\section{Therapeutic potency of the TSPO ligands}

Beneficial effects of TSPO ligands on neurodegenerative disease and anxiety disorders have been reported in various experimental models. Five-week administration of PK11195 in 3xTg-AD mice showed improved memory and reduced amyloid beta deposition in the hippocampus (36). XBD173, a synthetic ligand of the TSPO, ameliorated degeneration of dopaminergic neurons, preserved dopamine metabolism, and recovered motor dysfunction in MPTP-treated female mice, the model for PD (37). In experimental autoimmune encephalomyelitis (EAE), the model for multiple sclerosis (MS), administration of etifoxine before the onset of EAE reduced its severity, and administration at the peak of the disease progression improved symptomatic recovery (38).

Anxiolytic effects of TSPO ligands were also reported in various experimental models. In adrenalectomized and castrated rats, etifoxine increased the local steroids levels in the brain, such as allopregnanlone, the positive modulator of the $\mathrm{GABA}_{\mathrm{A}}$, and had anxiolytic effects (39). Other TSPO ligands, DAA1097 and DAA1106, also promoted steroidogenesis from mitochondria and had anxiolytic effects in anxiety behavior tests, such as the light/dark exploration test and elevate plus maze test (40). In an inflammatory pain model, acetamide (ZBD-2) reduced the anxiety-like behaviors and prevented an imbalance of excitatory and inhibitory transmission in the basolateral amygdala (BLA) synapses (41),suggesting that TSPO ligands have anxiolytic effects through various mechanisms.

The main mechanisms underlying the beneficial effects of the TSPO ligand in various experimental models of neurodegenerative conditions are likely to result from amelioration of microglia activation and neuroinflammation (42).

\section{Challenge of the role of TSPO in the neuroinflammation}

TSPO is now widely used as the marker for neuroinflammation or microglia activation, because its protein level is remarkably upregulated in activated glial cells, especially in microglia during neural disorders or injury (43). Furthermore, the anti-inflammatory effects of TSPO ligands were identified in both the peripheral nervous system and the CNS $(34,44,45)$.
The biological action of the TSPO protein itself under neuroinflammatory conditions was also examined. For instance, knockdown of TSPO augmented the inflammatory response to lipopolysaccharide (LPS), whereas overexpression of TSPO led to opposite effects (46-48). Therefore, an increase in TSPO level during inflammation was regarded as the adaptive response that may restrict an aggressive inflammatory response. However, there are several points worthy of attention regarding the relation of TSPO to neuroinflammation.

First, TSPO signaling studies have been mostly restricted to activated microglia, which are the resident macrophage in the brain that is the master regulator of the brain's immune response. Under normal condition, the brain is barely affected by the peripheral immune system. However, under pathological conditions, peripheral immune cells (e.g., T cells, B cells, and monocytes) infiltrate the brain toward the damaged area (49, 50). Neurons, astrocytes, and endothelial cells express immune checkpoint genes and regulate systemic immune activity in the brain, modulating the function of microglia (49, 50). In the light of this view, altered TSPO signaling should be understood as the result of systemic change through interaction between various cell types involved in neuroimmune modulation and be examined, including astrocytes, endothelial cells, or neurons depending on the region, as well as microglia. This point is especially important in the diseases involving low-grade inflammation. For instance, in experimental models and human patients of schizophrenia, lowered prefrontal TSPO expression was associated with behavior abnormalities and increased levels of cytokines. Intriguingly, altered TSPO levels in a schizophrenia model were not restricted to microglia, but were observed in other cell types, such as astrocytes and vascular endothelial cells (51). These data suggest the need for systemic profiling of inflammation and characterization of other inflammatory markers when the TSPO level is interpreted.

Another point concerning the role of TSPO is that altered TSPO expression not only represents the inflammatory response, but also signifies the aberration in cellular metabolism, energy homeostasis, or oxidative stress during inflammation (35). Therefore, correlation of altered TSPO levels with microglia activation can be the circumstantial aftermath of, rather than represent, direct causality with neuroinflammation.

The last point is that the role of TSPO in inflammation may be quite different depending on the species. Recent research on rodent and human microglia showed that basal TSPO gene expression was higher in human monocyte-derived macrophages (MDM) than in human adult microglia (HAM). Neither IFN$\gamma /$ LPS (a classic pro-inflammatory stimulus) nor IL4/IL13 (a stimulus of reparative activation) increased TSPO gene expression in HAM. In contrast, pro-inflammatory activation of human MDM reduced TSPO expression. However, as observed previously, those immune stimuli increased TSPO expression in a rodent's monocyte-derived macrophages (MDM) and primary microglia. These results indicate that increased TSPO 
expression in human positron-emission tomography (PET) imaging may indicate an increased number of microglia or monocyte-derived macrophages rather than microglia activation per se, emphasizing the cautious interpretation of altered TSPO levels (52).

\section{Complex functions of TSPO in the CNS}

Now a TSPO-floxed mouse is available and conditional knockout of TSPO using Cre-LoxP system can be conducted. The hGFAP-driven conditional TSPO knockout resulted in the deletion of TSPO in the CNS, while microglial TSPO expression was spared (53). The neural lineage-specific TSPO knockout mice showed a decrease in astrogliosis in response to neural injury in EAE, a popular animal model of MS. This decline in astrogliosis was associated with lessening the severity of $E A E$, indicating a functional role for TSPO in activation of astrocytes (53).

A recent CRISPR-Cas9-mediated TSPO gene knockout study in human primary microglia reported that TSPO in human microglia is not related to steroidogenesis but to alterations in MMP, cytosolic $\mathrm{Ca}^{2+}$, and VDAC expression (54).

\section{TSPO ligands as diagnostic tools: is TSPO a true target?}

TSPO PET imaging has been developed to detect the inflammatory response as a non-invasive molecular imaging tool used to diagnose and manage CNS disorders. Radio-labeled RO5-4864 (benzodiazepine derivative) and PK11195 (isoquinoline carboxamide derivative) are the first generated probes to be used in PET imaging. For Ro5-4864, it has the problem that its binding affinity can be changed by temperature and species; so the initial studies on TSPO PET imaging mainly used [11C]PK11195 (55). [11C]PK11195 is the most studied ligand and is used as the standard in validation of other TSPO ligands in PET imaging. Early in the study, [11C]PK11195 was successfully employed for the study of several neurodegenerative diseases, such as MS, AD, PD, and amyotrophic lateral sclerosis (ALS) (56-59). Especially in AD, [11C]PK11195 binding affinity highly correlates with the degree of disease progression (57). However, a high level of nonspecific binding of PK11195 resulting from its lipophilic nature required the development of new ligands that have a higher affinity to TSPO and less non-specific binding.

A second generation of TSPO ligands based on the indole-ring structure of oracetamidobenzoxazolone was made to improve their binding affinity and specificity to TSPO. [11C]SSR180575 is the most-studied new ligand with higher imaging contrast than [11C]PK11195 has (60). Surprisingly, TSPO radio-ligands of the second generation showed the existence of binding targets other than TSPO, such as human constitutive androstane receptor (hCAR) but not mouse CAR (mCAR) (Fig. 2) (61). CAR is the transcription factor that regulates the expression of several transport proteins and cytochrome P450, Therefore, the CAR protein can modulate drug metabolism and excretion for a cellular defense system against xenobiotic insults. The CAR protein is also involved in various cell functions, including cellular metabolism and steroid hormone response. Interestingly, human TSPO plays no role in the PK11195 inhibition of hCAR activity, because knockdown of TSPO in HeLa cells did not alter PK11195 effects on hCAR. This finding indicates that TSPO ligands may have different targets depending on species, and proteins other than TSPO can play roles in mediating the pharmaceutical effects of TSPO ligands (61).

In conclusion, PET imaging with TSPO ligands has correlation with CNS disorders, but increased TSPO expression does not necessarily indicate microglia activation. Instead, increased TSPO ligand binding may indicate increased binding targets other than TSPO or increased immune-cell populations in certain contexts.

\section{Clinical trials on TSPO ligands}

Ligands that are known to have binding affinity to TSPO, such as TRO19622 (Olesoxime), TRO40303, etofoxine, and XBD173, have entered clinical trial phase II or III. TRO19622 (Olesoxime) is the potential drug for spinal muscular atrophy (SMA) and ALS. It promoted motor-neuron survival in vitro. In a SOD1 ${ }^{\mathrm{G} 93 \mathrm{~A}}$ transgenic mice model for ALS, TRO19622 improved motor performance, delayed the onset of the clinical disease, and extended the survival period (62). TRO19622 has a cholesterol-like structure and can bind to the CRAC domain of TSPO with a Ki value of $100 \mathrm{nM}$. However, it also binds to the neurosteroid binding site of VDAC. The assumed molecular mechanism by which TRO19622 affects SMA is by targeting mPTP opening and cytochrome c release (62).

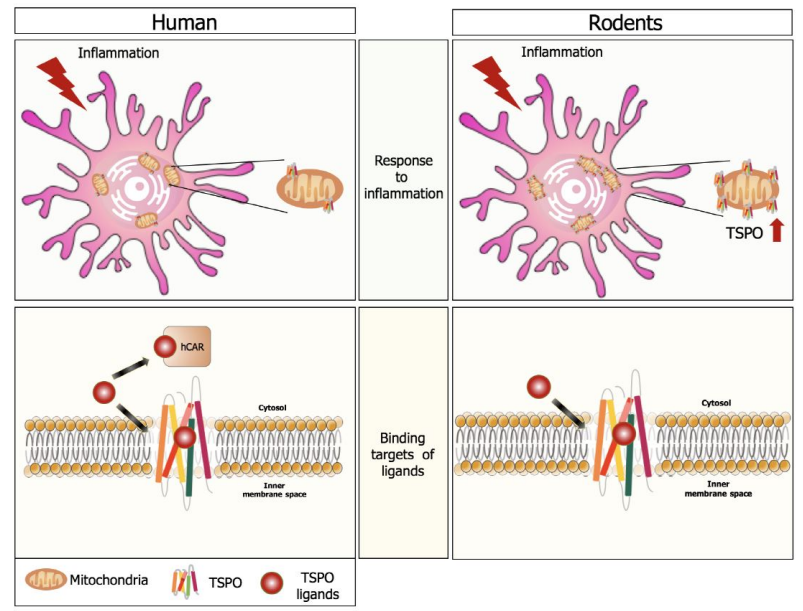

Fig. 2. Differences between human and rodent TSPO. Unlike the positive correlation between TSPO and neuroinflammation in rodents, TSPO expression in human is not related to inflammation level. Binding partners of TSPO ligands are also different between the two species. For instance, PK11195 has a binding affinity to human CAR but not to mouse CAR. 
TRO40303 is the cardio-protective compound that has a cholesterol-like structure. This compound was derived from the optimization process for TRO19622 and affects mPTP opening, $\mathrm{Ca}^{2+}$ overload, ROS generation, and MMP (63). Along with TRO19622, it also binds at the CRAC site of TSPO and is now in clinical trial phase II as a drug for reducing reperfusion injury in patients.

Etifoxine is the compound that has entered clinical trial III for treating anxiety in humans (64). Additionally, this compound has neuroprotective and neuroregenerative activities. These effects are mediated through the $\gamma$-aminobutyric-acid receptor type $A(G A B A A)$ receptor and steroid biosynthesis. Especially, increased steroid biosynthesis is a remarkable feature after etifoxine treatment, which in $1 \mathrm{~h}$ increased pregenelone, progesterone and corticosterone in adrenal glands (65).

XBD173 (Emapunil) is another compound in clinical phase II with anxiolytic and anti-panic effects. It increased GABAmediated neurotransmission and counteracted panic attacks induced in rodents without sedation and tolerance development (66). Furthermore, XBD173 has neuroprotective and antiinflammatory actions in choroidal endothelial cells (67).

The neuroprotective effects of so-called TSPO ligands have been well documented. However, the biological mechanisms by which these ligands exert these pharmacological efficacies still remain elusive. The proposed target pathways are steroidogenesis, mPTP, intracellular $\mathrm{Ca}^{2+}$ signaling, ROS generation, and MMP, commonly modulating neuroinflammation through TSPO or together with its partners, such as VDAC. However, as shown below, the biological action mechanism of TSPO in the aforementioned pathways is being discounted.

\section{GENETIC STUDIES OF TSPO}

The early report of the embryonic lethality of the conventional TSPO knockout mice was considered to be evidence for the essential role of TSPO, especially in steroidogenesis (25). However, genetic studies on TSPO have recently resumed with unexpectedly striking results.

\section{Discrepancy between genetic and pharmacological studies of TSPO}

In contrast to the previous report of the embryonic lethality of global TSPO-deleted $\left(T_{s p o^{-/}}\right)$mice, recently generated $T s p o^{-/-}$ mice were born and developed normally. The mice did not show discernible phenotypes, including abnormality in steroid hormones generation $(68,69)$. Therefore, observations with new $\mathrm{Tspo}^{-/}$mice argue against the most-cited biological role of TSPO, mitochondrial cholesterol import for steroidogenesis.

Similarly, different TSPO knockout mice in which the Tspo gene was conditionally deleted in the liver and heart disputed the roles of TSPO in mPTP formation, erythropoiesis, heme biosynthesis, and porphyrin-mediated phototoxic cell death, all of which were previously purported functions of TSPO (70, 71). These results of recent genetic studies are inconsistent with those of the pharmacological studies conducted for decades, prompting our change in the direction of TSPO study.

At the cellular level, CRISPR/Cas-9-mediated Tspo deletion in MA-10 cells also revealed that Tspo deletion did not affect steroidogenesis production (72). CRISPR-Cas9-mediated knockout and lentiviral knockdown of the TSPO gene was also done in human microglia C2 cells (54). This study investigated the involvement of TSPO in the steroid synthesis, MMP, $\mathrm{Ca}^{2+}$ uptake, and respiration. Notably, it examined the effects of different TSPO levels by comparing TSPO knockdown and knockout cells. Interestingly, basal pregneneolone production was not changed by TSPO knockdown or knockout, and TSPO ligands that have steroidogenic effects also failed to induce pregnenolone production in both WT and Tspo knockout microglia. These data indicate that human microglia TSPO is not engaged in microglia steroidogenesis (54).

On the other hand, TSPO gene deletion resulted in significant reduction of MMP and increased cytosolic $\mathrm{Ca}^{2+}$ levels. However, the MMP and cytosolic $\mathrm{Ca}^{2+}$ level in TSPO knockdown microglia was not changed. Interestingly, deletion of the TSPO gene led to decreased VDAC1 expression, supporting the relationship between TSPO-VDAC interaction. In addition, the oxygen consumption rate was checked and showed that TSPO deletion in human microglia reduced respiration but TSPO knockdown does not affect it, suggesting that there are differences in phenotypes between TSPO knockdown and knockout (54). In conclusion, functional characterization of TSPO knockdown and knockout human microglia again discounts the essential role of TSPO in steroidogenesis and other presumed functions of TSPO.

\section{Perspectives for the future study of TSPO}

TSPO ligand-based studies presumed that the effects of the ligands are likely to be mediated by TSPO because they have high affinity to this protein. However, investigation with a new generation of the TSPO probes suggests the existence of targets other than TSPO $(61,62)$. Furthermore, recent reports propose that these ligands also can incorporate the lipid bilayer of mitochondria rather than targeting mitochondrial membrane proteins (73). It is clear that TSPO and its ligands have different mechanisms of action, and future studies should be aimed at identifying the authentic molecular targets of the ligands and, at the same time, at characterizing their effects more in depth, so that we can distinguish the roles of TSPO itself and its ligands (74).

The second point is the lack of understanding of the difference roles of TSPO in different species, especially in humans and murine models. Historically, TSPO has been regarded as a surrogate for the state of inflammation with its remarked upregulation under neuroinflammatory conditions. However, recent studies indicate that increased TSPO binding sites in human PET imaging has implications different from those of increased TSPO expression in rodents. These recent 
findings may imply aspects of brain homeostasis that are different between humans and rodents $(52,57,61)$.

Future study on the role of TSPO in neurological diseases should also keep in mind that, depending on the grades of inflammation, correlations of TSPO with neuroinflammation should be interpreted with caution, and other inflammatory components should be considered together. In human schizophrenia, which shows increased inflammatory cytokine expression but with low grade of neuroinflammation, TSPO expression in the prefrontal area was reduced, not increased (51). The potency of TSPO PET imaging as a diagnostic tool cannot be refuted. However, to understand the biological and pathophysiological role of TSPO, a general understanding of TSPO and inflammation should be reconsidered.

The last point is the need for thorough genetic studies of TSPO under both normal physiological and pathological conditions. In spite of the past several decades of research, the TSPO field is facing an unexpected twist. To advance the field further, we need to understand the differences between the actions of ligands and of the protein itself, recognize species differences, discover other previously unrecognized functions of TSPO through genetic modulation, and identify the true targets of the so-called TSPO binding drugs.

\section{ACKNOWLEDGEMENTS}

This work was supported by the National Research Foundation of Korea (NRF) grant (2018M3C7A1056275) and the Korean Brain Research Institute basic research program (19-BR-04-02) of the Ministry of Science and ICT of Korea.

\section{CONFLICTS OF INTEREST}

The authors have no conflicting interests.

\section{REFERENCES}

1. Wallace DC (2012) Mitochondria and cancer. Nat Rev Cancer 12, 685

2. Green DR, Galluzzi L and Kroemer G (2011) Mitochondria and the autophagy-inflammation-cell death axis in organismal aging. Science 333, 1109-1112

3. Chen H and Chan DC (2009) Mitochondrial dynamicsfusion, fission, movement, and mitophagy-in neurodegenerative diseases. Hum Mol Genet 18, R169-R176

4. Pfanner N, Warscheid B and Wiedemann N (2019) Mitochondrial proteins: from biogenesis to functional networks. Nat Rev Mol Cell Biol 20, 267-284

5. Braestrup C, Albrechtsen R and Squires RF (1977) High densities of benzodiazepine receptors in human cortical areas. Nature 269, 702-704

6. Papadopoulos V (1998) Structure and function of the peripheral-type benzodiazepine receptor in steroidogenic cells. Proc Soc Exp Biol Med 217, 130-142

7. Azarashvili T, Krestinina O, Yurkov I, Evtodienko $\mathrm{Y}$ and
Reiser G (2005) High-affinity peripheral benzodiazepine receptor ligand, PK11195, regulates protein phosphorylation in rat brain mitochondria under control of $\mathrm{Ca} 2+$. J Neurochem 94, 1054-1062

8. Hirsch JD, Beyer CF, Malkowitz L, Beer B and Blume AJ (1989) Mitochondrial benzodiazepine receptors mediate inhibition of mitochondrial respiratory control. Mol Pharm 35, 157-163

9. Hirsch T, Decaudin D, Susin SA et al (1998) PK11195, a ligand of the mitochondrial benzodiazepine receptor, facilitates the induction of apoptosis and reverses Bcl-2-mediated cytoprotection. Exp Cell Res 241, 426-434

10. Lee D, Kang S, Lee R et al (2004) Effects of peripheral benzodiazepine receptor ligands on proliferation and differentiation of human mesenchymal stem cells. J Cell Physiol 198, 91-99

11. Kim T and Pae AN (2016) Translocator protein (TSPO) ligands for the diagnosis or treatment of neurodegenerative diseases: A patent review (2010-2015; part 1). Expert Opin Ther Pat 26, 1325-1351

12. Fan J, Lindemann $P, G$ J Feuilloley $M$ and Papadopoulos $V$ (2012) Structural and functional evolution of the translocator protein (18 kDa). Curr Mol Med 12, 369-386

13. Yeliseev AA, Krueger KE and Kaplan S (1997) A mammalian mitochondrial drug receptor functions as a bacterial "oxygen" sensor. Proc Natl Acad Sci U S A 94, 5101-5106

14. Chapalain A, Chevalier S, Orange N, Murillo L, Papadopoulos $\mathrm{V}$ and Feuilloley MG (2009) Bacterial ortholog of mammalian translocator protein (TSPO) with virulence regulating activity. PLoS One 4, e6096

15. Riond J, Leplatois $P$, Laurent $P$ et al (1991) Expression and pharmacological characterization of the human peripheraltype benzodiazepine receptor in yeast. Eur J Pharmacol 208, 307-312

16. Fan J, Rone MB and Papadopoulos V (2009) Translocator protein 2 is involved in cholesterol redistribution during erythropoiesis. J Biol Chem 284, 30484-30497

17. Li F, Liu J, Zheng Y, Garavito RM and Ferguson-Miller S (2015) Crystal structures of translocator protein (TSPO) and mutant mimic of a human polymorphism. Science 347 555-558

18. Jaremko $\measuredangle$, Jaremko $M$, Giller $K$, Becker $S$ and Zweckstetter M (2014) Structure of the mitochondrial translocator protein in complex with a diagnostic ligand. Science 343, 13631366

19. Guo Y, Kalathur RC, Liu Q et al (2015) Structure and activity of tryptophan-rich TSPO proteins. Science 347, 551-555

20. Veenman L, Vainshtein A, Yasin N, Azrad M and Gavish $M$ (2016) Tetrapyrroles as endogenous TSPO ligands in eukaryotes and prokaryotes: Comparisons with synthetic ligands. Int J Mol Sci 17, 880

21. Owen DR, Yeo AJ, Gunn RN et al (2012) An 18-kDa translocator protein (TSPO) polymorphism explains differences in binding affinity of the PET radioligand PBR28. J Cereb Blood Flow Metab 32, 1-5

22. Toor JS and Sikka SC (2017) Developmental and Reproductive Disorders - Role of Endocrine Disruptors in Testicular Toxicity; in Rep Dev Toxicol 1111-1121, 
Elsevier,

23. Papadopoulos V, Mukhin A, Costa E and Krueger K (1990) The peripheral-type benzodiazepine receptor is functionally linked to Leydig cell steroidogenesis. J Bioll Chem 265, 3772-3779

24. Chung J-Y, Chen H, Midzak A, Burnett A, Papadopoulos $V$ and Zirkin BR (2013) Drug ligand-induced activation of translocator protein (TSPO) stimulates steroid production by aged brown Norway rat Leydig cells. Endocrinology 154, 2156-2165

25. Papadopoulos V, Amri H, Li H, Boujrad N, Vidic B and Garnier M (1997) Targeted disruption of the peripheral-type benzodiazepine receptor gene inhibits steroidogenesis in the R2C Leydig tumor cell line. J Biol Chem 272, 3212932135

26. Hauet T, Yao ZX, Bose HS et al (2005) Peripheral-type benzodiazepine receptor-mediated action of steroidogenic acute regulatory protein on cholesterol entry into leydig cell mitochondria. Mol Endocrinol 19, 540-554

27. McEnery MW, Snowman AM, Trifiletti RR and Snyder SH (1992) Isolation of the mitochondrial benzodiazepine receptor: association with the voltage-dependent anion channel and the adenine nucleotide carrier. Proc Natl Acad Sci U S A 89, 3170-3174

28. Büki A, Okonkwo DO, Wang KK and Povlishock JT (2000) Cytochrome c release and caspase activation in traumatic axonal injury. J Neurosci 20, 2825-2834

29. Lemasters JJ, Theruvath TP, Zhong $Z$ and Nieminen A-L (2009) Mitochondrial calcium and the permeability transition in cell death. Biochim Biophys Acta Bioenerg 1787, 1395-1401

30. Azarashvili T, Krestinina O, Baburina Y et al (2015) Combined effect of G3139 and TSPO ligands on Ca2+-induced permeability transition in rat brain mitochondria. Arch Biochem Biophys 587, 70-77

31. Shoshan-Barmatz V, Pittala S and Mizrachi D (2019) VDAC1 and the TSPO: Expression, Interactions, and Associated Functions in Health and Disease States. Int J Mol Sci 20, 3348

32. Selvaraj $V$ and Stocco DM (2015) The changing landscape in translocator protein (TSPO) function. Trends Endocrinol Metab 26, 341-348

33. Kim S, Kim N, Park S et al (2019) Tanycytic TSPO inhibition induces lipophagy to regulate lipid metabolism and improve energy balance. Autophagy [Epub ahead of print]

34. Lee JW, Nam H and Yu SW (2016) Systematic analysis of translocator protein $18 \mathrm{kDa}$ (TSPO) ligands on toll-like receptors-mediated pro-inflammatory responses in microglia and astrocytes. Exp Neurobiol 25, 262-268

35. Notter T, Coughlin JM, Sawa A and Meyer U (2018) Reconceptualization of translocator protein as a biomarker of neuroinflammation in psychiatry. Mol Psychiatry 23, 36

36. Christensen A and Pike CJ (2018) TSPO ligand PK11195 improves Alzheimer-related outcomes in aged female 3xTg-AD mice. Neurosci Lett 683, 7-12

37. Gong J, Szego ÉM, Leonov A et al (2019) Translocator protein ligand protects against neurodegeneration in the MPTP mouse model of Parkinsonism. J Neurosci 39, 37523769

38. Daugherty DJ, Selvaraj V, Chechneva OV, Liu XB,
Pleasure DE and Deng W (2013) A TSPO ligand is protective in a mouse model of multiple sclerosis. EMBO Mol Med 5, 891-903

39. Verleye M, Akwa Y, Liere P et al (2005) The anxiolytic etifoxine activates the peripheral benzodiazepine receptor and increases the neurosteroid levels in rat brain. Pharmacol Biochem Behar 82, 712-720

40. Okuyama S, Chaki S, Yoshikawa R et al (1999) Neuropharmacological profile of peripheral benzodiazepine receptor agonists, DAA1097 and DAA1106. Life Sci 64, 1455-1464

41. Wang D-s, Tian Z, Guo Y-y et al (2015) Anxiolytic-like effects of translocator protein (TSPO) ligand ZBD-2 in an animal model of chronic Pain. Mol Pain 11, 16

42. Da Pozzo E, Giacomelli C, Barresi E et al (2015) Targeting the $18-\mathrm{kDa}$ translocator protein: recent perspectives for neuroprotection. Biochem Soc Trans 43, 559-565

43. Kim EJ and Yu SW (2015) Translocator protein 18 kDa (TSPO): old dogma, new mice, new structure, and new questions for neuroprotection. Neural Regen Res 10, 878

44. Kupa LdVK, Drewes CC, Barioni ED, Neves CL, Sampaio SC and Farsky SH (2017) Role of Translocator 18 KDa Ligands in the Activation of Leukotriene B4 Activated G-Protein Coupled Receptor and Toll Like Receptor-4 Pathways in Neutrophils. Front Pharmacol 8, 766

45. Lee JW, Kim LE, Shim HJ, Kim EK, Hwang WC and Yu S-W (2016) A translocator protein $18 \mathrm{kDa}$ ligand, Ro54864, inhibits ATP-induced NLRP3 inflammasome activation. Biochem Biophys Res Comm 474, 587-593

46. Wang W, Zhang L, Zhang X et al (2016) Lentiviralmediated overexpression of the $18 \mathrm{kDa}$ translocator protein (TSPO) in the hippocampal dentate gyrus ameliorates LPS-induced cognitive impairment in mice. Front Pharmacol 7, 384

47. Bae KR, Shim HJ, Balu D, Kim SR and Yu SW (2014) Translocator protein $18 \mathrm{kDa}$ negatively regulates inflammation in microglia. J Neuroimmune Pharmacol 9, 424-437

48. Wang $M$, Wang $X$, Zhao $L$ et al (2014) Macrogliamicroglia interactions via TSPO signaling regulates microglial activation in the mouse retina. J Neurosci 34, 37933806

49. Deczkowska A and Schwartz M (2018) Targeting neuroimmune communication in neurodegeneration: Challenges and opportunities. J Exp Med 215, 2702-2704

50. Schwartz M (2017) Can immunotherapy treat neurodegeneration? Science 357, 254-255

51. Notter T, Coughlin JM, Gschwind T et al (2018) Translational evaluation of translocator protein as a marker of neuroinflammation in schizophrenia. Mol Psychiatry 23, 323

52. Owen DR, Narayan N, Wells L et al (2017) Proinflammatory activation of primary microglia and macrophages increases $18 \mathrm{kDa}$ translocator protein expression in rodents but not humans. J Cereb Blood Flow Metab 37 , 2679-2690

53. Daugherty DJ, Chechneva O, Mayrhofer F and Deng W (2016) The hGFAP-driven conditional TSPO knockout is protective in a mouse model of multiple sclerosis. Sci Rep 6, 22556

54. Milenkovic VM, Slim D, Bader S et al (2019) CRISPR-Cas9 Mediated TSPO Gene Knockout alters Respiration and 
Cellular Metabolism in Human Primary Microglia Cells. Int J Mol Sci 20, 3359

55. Alam MM, Lee J and Lee S-Y (2017) Recent progress in the development of TSPO PET ligands for neuroinflammation imaging in neurological diseases. Nucl Med Mol Imaging 51, 283-296

56. Debruyne J, Versijpt J, Van Laere K et al (2003) PET visualization of microglia in multiple sclerosis patients using [11C] PK11195. Eur J Neurol 10, 257-264

57. Cagnin A, Brooks DJ, Kennedy AM et al (2001) In-vivo measurement of activated microglia in dementia. The Lancet 358, 461-467

58. Gerhard A, Pavese N, Hotton G et al (2006) In vivo imaging of microglial activation with [11C](R)-PK11195 PET in idiopathic Parkinson's disease. Neurobiol Dis 21, 404412

59. Turner M, Cagnin A, Turkheimer F et al (2004) Evidence of widespread cerebral microglial activation in amyotrophic lateral sclerosis: an [11C](R)-PK11195 positron emission tomography study. Neurobiol Dis 15, 601-609

60. Chauveau F, Boutin H, Van Camp $N$ et al (2011) In vivo imaging of neuroinflammation in the rodent brain with [11 C] SSR180575, a novel indoleacetamide radioligand of the translocator protein (18 kDa). Eur J Nucl Med Mol Imaging 38, 509-514

61. Li L, Chen T, Stanton JD, Sueyoshi T, Negishi M and Wang $H$ (2008) The peripheral benzodiazepine receptor ligand 1-(2-chlorophenyl-methylpropyl)-3-isoquinoline-carboxamide is a novel antagonist of human constitutive androstane receptor. Mol Pharmacol 74, 443-453

62. Bordet T, Buisson B, Michaud M et al (2007) Identification and characterization of cholest-4-en-3-one, oxime (TRO19622), a novel drug candidate for amyotrophic lateral sclerosis. J Pharmacol Exp Ther 322, 709-720

63. Schaller S, Paradis S, Ngoh GA et al (2010) TRO40303, a new cardioprotective compound, inhibits mitochondrial permeability transition. J Pharmacol Exp Ther 333, 696706

64. Nguyen N, Fakra E, Pradel V et al (2006) Efficacy of etifoxine compared to lorazepam monotherapy in the treatment of patients with adjustment disorders with anxiety: a double-blind controlled study in general practice. Hum Psychopharmacol 21, 139-149

65. do Rego JL, Vaudry D and Vaudry H (2015) The nonbenzodiazepine anxiolytic drug etifoxine causes a rapid, receptor-independent stimulation of neurosteroid biosynthesis. PLoS One 10, e0120473

66. Rupprecht R, Rammes G, Eser D et al (2009) Translocator protein $(18 \mathrm{kD})$ as target for anxiolytics without benzodiazepine-like side effects. Science 325, 490-493

67. Biswas L, Farhan F, Reilly I, Bartholomew C and Shu X (2018) TSPO Ligands Promote Cholesterol Efflux and Suppress Oxidative Stress and Inflammation in Choroidal Endothelial Cells. Int J Mol Sci 19, 3740

68. Tu LN, Morohaku K, Manna PR et al (2014) Peripheral benzodiazepine receptor/translocator protein global knockout mice are viable with no effects on steroid hormone biosynthesis. J Biol Chem 289, 27444-27454

69. Morohaku K, Pelton SH, Daugherty DJ, Butler WR, Deng W and Selvaraj V (2014) Translocator protein/peripheral benzodiazepine receptor is not required for steroid hormone biosynthesis. Endocrinology 155, 89-97

70. Šileikytė J, Blachly-Dyson E, Sewell R et al (2014) Regulation of the mitochondrial permeability transition pore by the outer membrane does not involve the peripheral benzodiazepine receptor (Translocator Protein of $18 \mathrm{kDa}$ (TSPO)). J Biol Chem 289, 13769-13781

71. Zhao AH, Tu LN, Mukai C et al (2016) Mitochondrial translocator protein (TSPO) function is not essential for heme biosynthesis. J Biol Chem 291, 1591-1603

72. Tu LN, Zhao AH, Stocco DM and Selvaraj V (2015) PK11195 effect on steroidogenesis is not mediated through the translocator protein (TSPO). Endocrinology 156, 10331039

73. Hatty CR, Le Brun AP, Lake V et al (2014) Investigating the interactions of the $18 \mathrm{kDa}$ translocator protein and its ligand PK11195 in planar lipid bilayers. Biochim Biophys Acta Biomembr 1838, 1019-1030

74. Bader S, Wolf L, Milenkovic VM et al (2019) Differential effects of TSPO ligands on mitochondrial function in mouse microglia cells. Psychoneuroendocrinology 106, 65-76 